\title{
Quantum Entanglement via Superradiance of a Bose-Einstein Condensate ${ }^{1}$
}

\author{
M. E. Taşgın ${ }^{a, *}$, M. Ö. Oktel ${ }^{b}$, L. You ${ }^{c}$, and Ö. E. Müstecaplığ̆lu ${ }^{c, * *}$ \\ ${ }^{a}$ Department of Physics, Koç University, 34450 Sartyer, Istanbul, Turkey \\ ${ }^{b}$ Department of Physics, Bilkent University, 06800 Bilkent, Ankara, Turkey \\ ${ }^{c}$ School of Physics, Georgia Institute of Technology, Atlanta, Georgia 30332, USA \\ *e-mail: metasgin@ku.edu.tr \\ **e-mail: omustecap@ku.edu.tr
}

Received October 2, 2009; published online February 2, 2010

\begin{abstract}
We adopt the coherence and built-in swap mechanism in sequential superradiance as a tool for obtaining continuous-variable (electric/magnetic fields) quantum entanglement of two counter-propagating pulses emitted from the two end-fire modes. In the first-sequence, end-fire modes are entangled with the side modes. In the second sequence, this entanglement is swapped to in between the two opposite end-fire modes. Additionally, we also examine the photon number correlations. No quantum correlations is observed in this variable.
\end{abstract}

DOI: $10.1134 / \mathrm{S} 1054660 X 10050191$

\section{INTRODUCTION}

Superradiance (SR), first predicted by Dicke [1] in 1954 and first experimentally demonstrated near the optical region in 1973 by Skribanowitz et al. [2], refers to the collective spontaneous emission of an ensemble of atoms. Since the phases of atoms cohere, multiatomic ensemble displays a macroscopic dipole moment that is proportional to the number of atoms, $N$. This gives rise to emission intensity proportional to the square of the number of atoms, $\sim N^{2}$. This resembles the intensity of overlap of $N$ identical waves, all have the same (or very close) phase. In a normal or stimulated radiator $N$ atoms radiate independently, giving rise to intensity proportional to $N$. In order to be able to observe the collective radiation, pumping time must be smaller than the decoherence time $\left(T_{2}\right)$ [3] of the atomic phases.

In SR, atomic coherence induces spontaneously due to the interaction of all atoms with the common radiation field; spontaneous emission field [4]. In other collective phenomenon, such that free induction, photon echo and self-induced transparency [5, $6]$, intensity is also proportional to $N^{2}$. The phasing between the atoms, however, is established via the coherent pumping of the atoms to the excited state.

SR occurs in many systems [7], from thermal gases of excited atoms [8] and molecules [2], quantum dots and quantum wires [9-11], to Rydberg gases [12], and molecular nanomagnets [13]. On the other hand, however, SR in an elongated Bose-Einstein condensates (BECs) [14, 15] displays peculiar features. Due to the cooperative nature of SR, condensate atoms

\footnotetext{
${ }^{1}$ The article is published in the original.
}

$(\mathbf{p}=0)$ are scattered into higher momentum states collectively. And, because of the strong directionality of the end-fire mode radiation, atoms are scattered approximately to the same momentum mode. These are called side modes. Furthermore, atoms are recoiled to side modes phase consistently due to the collectivity. When a side mode is sufficiently occupied they also give rise to superradiant scattering and form new side modes. The resulting picture, Fig. 1, is a fanlike pattern of the side modes. This phenomenon, that is observed only in BEC sample [14], is called the sequential Superradiance.

After the demonstration of SR in BEC [14], serious efforts have been directed towards the study of quantum entanglement between condensed atoms and SR light pulses [16-18]. Entanglement between atoms through SR [9] is also studied. Possibility of quantum teleportation in quantum dots via SR [9] is proposed as a promising application.

Recently, SR was observed in the Kapitza-Dirac regime [19] with short pulse pump scheme. Rather than the fanlike pattern of longer pulse pump scheme [14], momentum side modes display X-shaped pattern in the Kapitza-Dirac regime. Backscattering of the side modes are observable only in the short time intervals, since energy is not conserved in the occupation of these modes. It is predicted, in this regime, that SR pulses must contain quantum entangled counterpropagating photons from the end-fire modes [20]. The origin of the quantum entanglement is the fourwave mixing of two atomic fields (forward and backward scattered side modes) with the two photonic fields (counter-propagating end-fire modes) [20]. The predicted form of interaction, containing the terms of 


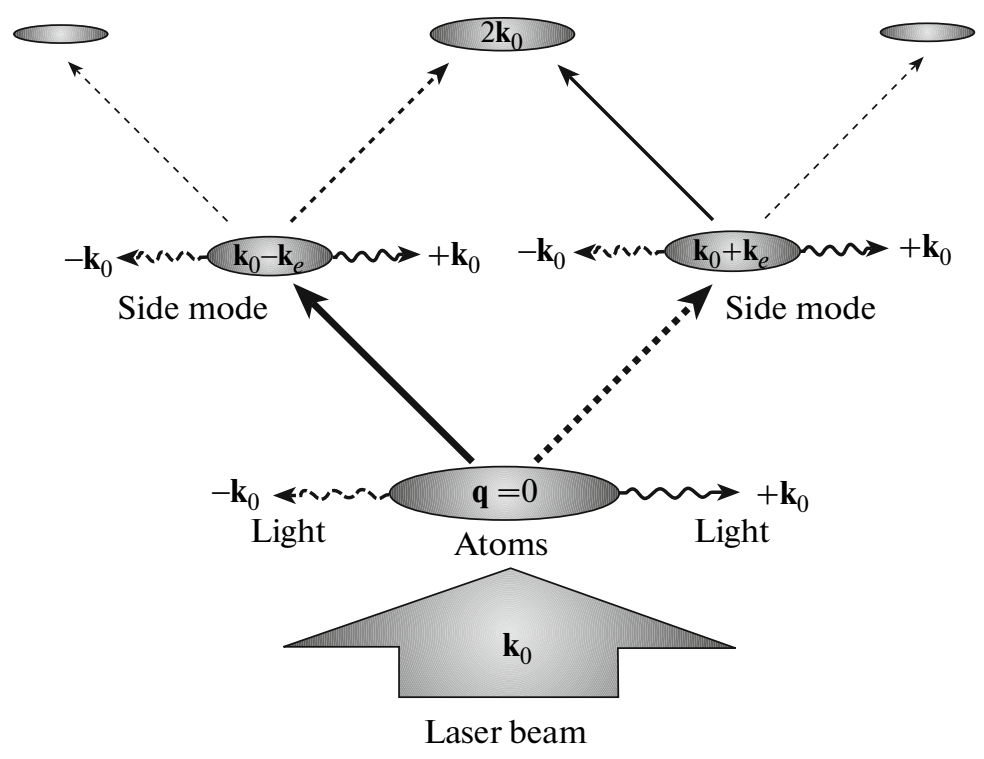

Fig. 1. (Color online) A fan-like atomic side mode pattern up to second order sequential superradiant scattering.

two-mode squeezing, suggests a continuous-variable (CV) entanglement in between the end-fire modes.

Experimental demonstration of quantum teleportation [21], via CV quantum entanglement of field amplitudes of two pulses, aroused great interest over the systems displaying CV correlations [22-25]. CV entanglement is adopted for quantum cryptology [26] as well as quantum computation [27-30]. Quantum computation is based upon the transfer of the entanglement between photon-photon, atom-atom and atom-photon pairs. The need for more durable entanglement drives the research on more correlated systems.

In this paper, we demonstrate the continuous-variable (CV) quantum entanglement of the two end-fire modes, during the sequential SR, even for a continuouswave pumped condensate [14]. The origins of the photon-photon entanglement within the fan-like pattern, however, is quite different than the predicted one (fourwave mixing) within the X-shaped pattern $[19,20]$.

The atom-photon entanglement generated in the first sequence of SR is swapped into the photon-photon entanglement in the second sequence. This is because; the side mode interacted with the rightwardpropagating end-fire mode in the first sequence, interacts with the leftward-propagating end-fire mode in the second sequence. In other words, counter-propagating end-fire modes are entangled due to the interaction with the same side mode, at. different times. In quantum information language, entanglement is swap is a technique to entangle particles that never before interacted [31-34]. We clearly identify the swapping of the entanglement in between the two pairs (atomphoton and photon-photon) in both of our analytical and computational results.
Additionally, we investigate the photon number correlation functions introduced in [35] and discussed in $[36,37]$. We test the existence of quantum correlations via referring to the violation of Bell-type inequalities [38-43]. We demonstrate that the behavior of photon number correlations unparallels the CV entanglement.

The paper is organized as follows. In Section 2 we give the outline of the full second quantized effectiveHamiltonian for the present system. The details of the derivation is available in $[44,45]$. CV entanglement criteria is discussed in Section 3, over which the confirmation of the existence of quantum correlations is judged. In Section 4, we solve the effective Hamiltonian analytically, under parametric and steady state approximations. We provide an intuitive argument, for the entanglement swap mechanism, to explain how such a Hamiltonian can generate EPR pairs out of non-interacting photons. In Section 5, we present our numerical results; the temporal dynamics of the entanglement and the accompanying field and atomic populations. This helps to illustrate the swapping of atom-photon entanglement to the photon-photon entanglement. In Section 6, we compare the dynamics of the correlations in the photon number with the CV entanglement. Section 7 contains our conclusions.

\section{MODEL HAMILTONIAN}

We consider an elongated condensate, of length $L$ and width $W$, axially symmetric about the long direction of the $z$-axis. The pump laser with frequency $\omega_{0}$ is along the $y$-axis and linearly polarized in the $x$-axis and detuned from the atomic resonance by $\Delta$. Adiabatically eliminating the atomic excited state while keeping the pump laser quantum, not like in [46], an 